\title{
Bessere Ausbildung in der Narkose und Anästhesie!
}

Von Dr. Heinz Wohlgemuth in Berlin.

Seit den Tagen des noch in unsere Zeit hineinragenden Velpeau, von dem der resignierte Ausspruch stammt: „Eviter la douleur dans les opérations est une chimère qui n'est pas permise de poursuivre", hat die Kunst schmerzlos zu operieren einen Riesenweg zurückgelegt, der geradezu etwas Verblüffendes hat. Eine große Zahl von Narcotica und Methoden steht uns zur Verfügung, um

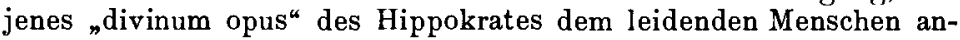
gedeihen zu lassen. Den größten Teil unserer Operationen können wir heute ohne allgemeine Narkose ausführen, und da, wo diese nötig ist. hat sie für uns durch die von mir eingeführte Sauerstoffchloroform- resp. Aethernarkose ihre Gefahren verloren.

Ist die Kunst der Anästhesie aber nun so einfach geworden, daß, wer will, nur eine Spritze zu nehmen braucht, die Cocainlösung suboder intrakutan in das Nervengewebe oder gar in den Medullarkanal zu bringen, um nun die gew ünschte Wirkung zu erzielen? Ist es nur nötig, den - wenn auch exakt wie kein Narkotiseur funktionierenden - Sauerstoffnarkoseapparat in Gang zu setzen, um eine tiefe, glatte, ungefährliche Narkose zu erzielen? -- Ebenso könnte jemand, der niemals eine größere Operation gemacht hat, eine Magen- oder Darmresektion oder eine Kehlkopfexstirpation mit Hoffnung auf ein gutes Resultat machen wollen, nachdem er sich aus dem Lehrbuch über die Art des Vorgehens informiert hat. So wenig wie das Zeugnis über das bestandene Staatsexamen dem jungen Arzt das Recht und die Zuversicht gibt, an lebenswichtigen Organen Operationen auszuführen, ohne daß er in mehrjährigem Studium mit besonderer Ausbildung sich die dazu notwendigen technischen und wissenschaftlichen Fähigkeiten angeeignet hat, so wenig ist dieser 
imstande, ohne besondere exakte Ausbildung eine örtliche oder allgemeine Anästhesie mit Aussicht auf Erfolg und ohne Gefahr für Leib und Leben des Kranken auszuführen. Für Leib und Leben! Denn die Narkose, sei sie Inhalations- oder Scopolamin-Morphiumnarkose, die Medullar- und in gewissen Grenzen auch die lokale Anästhesie ist und bleibt eine Gefahr, sie mögen so weit verbessert werden wie nur möglich. Sie werden stets eine Einverleibung von Giften in den menschlichen Körper bedeuten, die fast immer so groß ist, daß ihnen unter ungünstigen Umständen die Vitalität des Organismus erliegen kann. Niemals wird es eine absolut ungefährliche Narkose geben, wie es niemals eine absolut nngefährliche Operation gibt. Wie das gute Gelingen dieser, so ist die exakte und gefahrlose Ausführung jener eine Kunst, ein Können, das gelernt und geübt werden muß und von jedem, der als Arzt der Menschheit helfen, ihr nicht schaden will, gelernt und geübt sein sollte.

Kann denn der Kranke nicht verlangen, daß der, dem er seines Willens beraubt - Gesundheit und Leben anvertraut, Uebung und Erfahrung im Umgehen mit den das Leben unter Umständen gefährdenden Mitteln hat? Leistet ihm der Staat nicht in gewissem Sinne eine Garantie dafïr dadurch, daß er die Approbation als Arzt erteilt hat? -

Und nun sehe man sich die Narkosen an, die von Ungeïbten gemacht werden. Sie wissen wohl, da $\beta$ in der tiefen Narkose die Cornealreflexe erloschen sind. Und nun fangen sie an, knapp nach eingeleiteter Narkose mit dem Finger auf den Corneae - auf beiden natürlich! - herumzutrommeln, jede halbe, jede Viertelminute. Wie oft ist man versucht, ihnen die Worte Melchthals zuzurufen: „O, eine edle Himmelsgabe ist das Licht des Auges!" Was, wenn die Atmung nicht glatt ist, von diesen Helfern der Menschheit of mit dem Heisterschen Speculum, der Zungenzange und anderen Marterwerkzeugen gesündigt wird, das möchte wohl kein Operateur am eigenen Leibe jemals erfahren wollen.

Ich spreche nicht von den Narkosen in den großen Krankenanstalten und Kliniken, in denen ja die Famuli schließlich eine gewisse Routine erreichen, obgleich ich anch hier die unglaublichsten Dinge habe beobachten können. Schlimmer aber noch ist es, wenn in der Privatpraxis lokale Anästhesien versucht werden, die ,unempfindlichen " Patienten sich vor Schmerzen krümmen und nun, wenn gar keine operative Tätigkeit möglich ist, die allgemeine Narkose zur lokalen gefügt wird, zu den nicht unerheblichen Mengen Cocain das Chloroform, nun natürlich in großen Dosen, weil ja der Patient aufgeregt und viel schwerer in Narkose zu bringen ist. Ich will auch nicht davon reden, daß auch der Erfolg der Operation durch solche Narkosen resp. Anästhesien in Frage gestellt wird, aber eines muß man sich klar vor Augen halten, daß unter geeigneten Bedingungen bei einer solchen Narkose das Leben des $\mathrm{Pa}$ tienten zweifach bedroht ist, durch die Narkose an sich und durch die Unsicherheit, eine exakte Operation bis zur letzten Naht zll machen.

Gewi $\beta$ sind das alles Dinge, die fïr einen Operateur, in dessen Klinik die Narkosen und Anästhesien von einem durchaus geübten nnd den Ernst und die Wichtigkeit ihrer Funktion voll erfaßt habenden Narkotiseur gemacht werden, überwundene Zeiten bedeuten. Hier sind auch alle Hilfsmittel vorhanden, um sofort die Methode zu ändern, um, wenn es nottụt, die lokale Anästhesie mit der allgemeinen Narkose zu kombinieren und beim Gebrauch der Sauerstoff-Chloroformnarkose mit dem Minimum von Chloroform auszukommen. Doch das wichtigste sind die vorhandenen technischen Einrichtungen nicht. Die Kunst an sich muß geübt sein, die Kenntnis der Wirkungen der Narcotica, ihrer Zusammenwirkungen eine ausgebildete, das Auge und das Ohr des Narkotisenrs muß geschärft, seine Hand geäbt sein, will er den Kranken so schonen, wie dieser es erwartet und verlangen kann.

Wie wenige aber sind so geübt, in wie vielen öffentlichen und privaten Kliniken sind die Narkotiseure Vollassistenten, die in diesem "nebensächlichen“ Amt mit Ernst aufgehen! - In welcher Klinik, bei welchem Arzt sind Heister und Zungenzange nur noch historische Instrumente!

Die Lumbalanästhesie, Biers geniale Erfindung, möchte heute wohl kein Operateur mehr missen. Uneingeschränktes Lob verdient ihre Anwendung in den Fällen, in denen eine Inhalationsnarkose zn gefahrvoll erscheint. Wie nützlich könnte sie aber erst den Aerzten sein, die in einer kleinen Stadt oder auf dem Lande eine dringliche Operation zu machen gezwungen sind an Patienten, bei denen man a priori annehmen muß, da $\beta$ ihnen eine stundenlange Inhalationsnarkose die ohnehin schon flüchtige Spann- nnd Lebenskraft fast mit Sicherheit zu Boden drückt. Ich brauche nur an die Fälle von Ilens, Peritonitis, Stieldrehungen, Appendicitis und viele andere zu denken, die keinen Aufschub dulden, keinen Transport bis zur nächsten Stadt mit einem Krankenhaus, die keine allgemeine Narkose mehr vertragen. Garnicht zu sprechen davon, daß die lum. bale Anästhesie einen Assistenten erspart. Aber sie muß gelernt werden, ihre Technik, ihre Zufälligkeiten müssen ein solider Be- stand des Wissensschatzes dessen werden, der mit Ruhe und Umsicht und ohne Zagen an ihre Ausführung gehen und - ein gutes Resultat haben will.

Oft schon bin ich dafür eingetreten, diesem Zweige der medizinischen Kunst und Wissenschaft, der für den leidenden Menschen wahrlich nicht Nebensächliches bedentet, zu der ihm gebiihrenden Wertschätzung zu verhelfen. Da werden seit Jahren Statistiken über die verschiedenen Arten der Narkose und lokalen Anästhesie gemacht, und ziffernmäßig wird berechnet, bei welchem Mittel - die meisten Menschen sterben. Und doch ist die ganze Narkosenfrage so einfach zu lösen. Man sorge für eine griindliche Ansbildung der Studierenden in der allgemeinen und lokalen Anästhesie, man gebe den Krankenhäusern und Kliniken einen Assistenten, der sich nur mit der Anästhesie beschäftigt, einen Anästhetisten, nnd die Zufälligkeiten und Unglücksfälle in der Narkose werden auf ein Minimum herabsinken. Die Praktikantenscheine, die nötig sind, um zum Staatsexamen zugelassen zu werden, ver mehre man um den einen, mit dem der Student sich ausweisen muß, daß er eine größere Reihe von allgemeinen und lokalen Anästhesien selbständig ausgefïhrt hat. Er hat sicher die Wichtigkeit des Praktikantenscheins der Pockenschutzimpfung.

Ich stehe nicht vereinzelt mit meiner Forderung. Kliniker von Namen und Ruf haben diese Notwendigkeit seit langem anerkannt, und ich weise wiederholt daranf hin, $\mathrm{da} \beta$ andere Länder. Amerika, England lange, jetzt auch Italien, mit dem Beispiel in der Frkennung dieses Bedïrfnisses vorangegangen sind. Lassen sich.diese Kurse und Vorlesungen in den Studienplan nicht einreihen, so verlege man sie wenigstens in das praktische Jahr und in die Fortbildungskurse für Aerzte. Die in London eingerichteten Fortbildnngskurse fïhren jedesmal als erstes Unterrichtsfach Anästhesie und Narkose. In nicht geringem Maße wird man hierdurch zum Heile der leidenden Menschheit beigetragen zu haben mit Genngtunng sich sagen können.

Auf dem im September d. J. tagenden II. Internationalen Chirurgenkongre 3 in Brüssel steht das Thema Anästhesie nnd Narkose als besonders wichtig diesmal auf der Tagesordnung. Hoffen wir, daß die Verhandlungen dazu beitragen werden, daß in allen Ländern der Unterricht in der Anästhesie zu einem für die Studierenden obligatorischen gemacht wird. 